\title{
Drug-Drug Cocrystals Provide Significant Improvements of Drug Properties in Treatment with Progesterone
}

Chunyang Guo, ${ }^{\star}$ \& Qi Zhang, ${ }^{*}$ Bingqing Zhu, ${ }^{\star}$ Zaiyong Zhang, ${ }^{\star}$ Xiaoyu Ma, ${ }^{\|, ~}$ Wenjuan Dai, ${ }^{*}$ Xiaoyi Gong, ${ }^{*}$ Guobin Ren, ${ }^{*}$,,$\S$ Xuefeng Mei ${ }^{*}, *$

†Laboratory of Pharmaceutical Crystal Engineering \& Technology, School of

Pharmacy and ${ }^{\S}$ Shanghai Key Laboratory of New Drug Design, School of Pharmacy, East China University of Science and Technology, Shanghai, 200237, China

\$Pharmaceutical Analytical \& Solid-State Chemistry Research Center, Shanghai Institute of Materia Medica, Chinese Academy of Sciences, Shanghai 201203, China.

"University of Chinese Academy of Sciences, No. 19A Yuquan Road, Beijing 100049, China

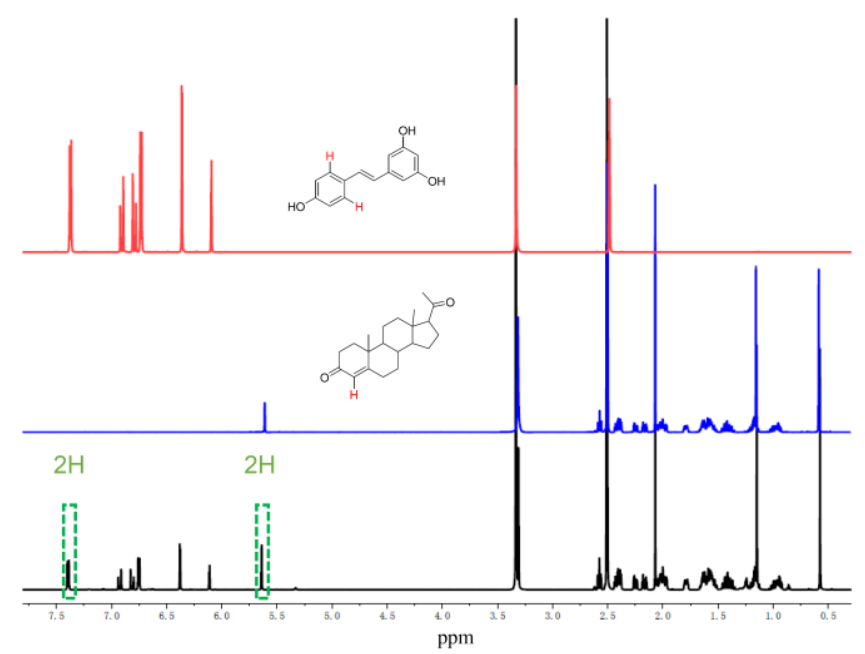


Figure S1. Comparison of ${ }^{1} \mathrm{H}-\mathrm{NMR}$ (C2D6SO, $400 \mathrm{MHZ}$ ) of RSV, Prog and ProgRSV cocrystal. The integration values suggested that the stoichiometry of Prog:RSV $=$ $2: 1$.

Table S1. List of H-bonding Lengths and Angles for Prog Cocrystals

\begin{tabular}{|c|c|c|c|c|c|}
\hline Form & Interactions & $\mathrm{H} \ldots \mathrm{A} / \AA$ & $\begin{array}{l}\mathrm{D} \ldots \mathrm{A} / \\
\AA\end{array}$ & $\begin{array}{l}<\mathrm{D}-\mathrm{H} \ldots \mathrm{A} / \\
\circ\end{array}$ & $\begin{array}{l}\text { Symmetry } \\
\text { Code }\end{array}$ \\
\hline \multirow[t]{4}{*}{$\begin{array}{l}\text { Prog- } \\
\text { SPF }\end{array}$} & $\mathrm{O} 3-\mathrm{H} 3 \cdots \mathrm{O} 1$ & 1.84 & 2.646 & 164 & $\begin{array}{l}1 / 2+x, 1 / 2-y \\
1-z\end{array}$ \\
\hline & $\mathrm{O} 4-\mathrm{H} 4 \mathrm{~A} \cdots \mathrm{O} 2$ & 1.95 & 2.765 & 169 & $\begin{array}{l}1 / 2+x, 1 / 2-y \\
-z\end{array}$ \\
\hline & $\mathrm{O} 5-\mathrm{H} 5 \cdots \mathrm{O} 3$ & 1.99 & 2.810 & 171 & $\begin{array}{l}1-x,-1 / 2+y \\
1 / 2-z\end{array}$ \\
\hline & $\mathrm{C} 16-\mathrm{H} 16 \mathrm{~A} \cdots \mathrm{O} 2$ & 2.48 & 2.885 & 104 & \\
\hline \multirow[t]{16}{*}{$\begin{array}{l}\text { Prog- } \\
\text { SPF-HH }\end{array}$} & $\mathrm{O} 3-\mathrm{H} 3 \cdots \mathrm{O} 2$ & 1.92 & 2.756 & 174 & $\begin{array}{l}1 / 2-x,-1 / 2+y, \\
-z\end{array}$ \\
\hline & O3A-H3A $\cdots$ O4A & 2.57 & 2.973 & 111 & $\mathrm{x}, \mathrm{y}, \mathrm{z}$ \\
\hline & O3A-H3A $\cdots$ O1A & 1.82 & 2.645 & 169 & $\begin{array}{l}1 / 2-x,-1 / 2+y, \\
-z\end{array}$ \\
\hline & $\mathrm{O} 4-\mathrm{H} 4 \mathrm{~B} \cdots \mathrm{O} 6$ & 2.15 & 2.985 & 173 & $\mathrm{x}, \mathrm{y}, \mathrm{z}$ \\
\hline & $\mathrm{O} 5-\mathrm{H} 5 \cdots \mathrm{O} 1$ & 1.83 & 2.667 & 175 & $1-x, y, 1-z$ \\
\hline & O5A-H5A $\cdots$ O6A & 2.09 & 2.606 & 120 & $\mathrm{x}, \mathrm{y}, \mathrm{z}$ \\
\hline & O5A-H5A $\cdots \mathrm{O} 8 \mathrm{~A}$ & 2.02 & 2.832 & 162 & $\mathrm{x},-1+\mathrm{y}, \mathrm{z}$ \\
\hline & 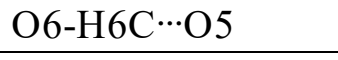 & 2.03 & 2.852 & 158 & $1-\mathrm{x},-1+\mathrm{y}, 1-\mathrm{z}$ \\
\hline & O4A-H4AA $\cdots$ O3A & 2.55 & 2.974 & 112 & $\mathrm{x}, \mathrm{y}, \mathrm{z}$ \\
\hline & 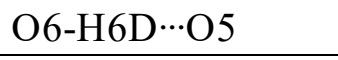 & 1.99 & 2.852 & 173 & $\mathrm{x},-1+\mathrm{y}, \mathrm{z}$ \\
\hline & O4A-H4AA $\cdots$ O1A & 1.84 & 2.674 & 171 & $\begin{array}{l}1 / 2-x, 1 / 2+y \\
-z\end{array}$ \\
\hline & O6A-H6AC $\cdots \mathrm{O} 2 \mathrm{~A}$ & 1.91 & 2.726 & 165 & $1-x, y, 1-z$ \\
\hline & O8A-H8AA $\cdots$ O3A & 2.38 & 2.823 & 113 & $\mathrm{x}, \mathrm{y}, \mathrm{z}$ \\
\hline & O8A-H8AA $\cdots$ O5A & 2.00 & 2.832 & 174 & $\mathrm{x}, 1+\mathrm{y}, \mathrm{z}$ \\
\hline & O8A-H8AA $\cdots$ O6A & 2.31 & 2.588 & 100 & $x, 1+y, \quad z$ \\
\hline & O7A-H7AC $\cdots \mathrm{O} 2 \mathrm{~A}$ & 1.84 & 2.675 & 172 & $1-x, y, 1-z$ \\
\hline \multirow{6}{*}{$\begin{array}{l}\text { Prog- } \\
\text { RSV-HH }\end{array}$} & $\mathrm{O} 5-\mathrm{H} 5 \cdots \mathrm{O} 3$ & 1.89 & 2.692 & 162 & $-1+x,-1+y, z$ \\
\hline & O6-H6 $\cdots$ O 8 & 1.83 & 2.633 & 163 & $-1+x, y, z$ \\
\hline & $\mathrm{O} 7-\mathrm{H} 7 \cdots \mathrm{O} 1$ & 1.88 & 2.675 & 159 & $1+x, 1+y, z$ \\
\hline & O8-H8A $\cdots \mathrm{O} 5$ & 1.96 & 2.670 & 139 & $\mathrm{x}, \mathrm{y}, \mathrm{z}$ \\
\hline & 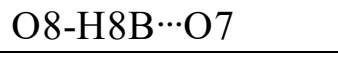 & 2.01 & 2.726 & 131 & $\mathrm{x},-1+\mathrm{y}, \mathrm{z}$ \\
\hline & $\mathrm{C} 2-\mathrm{H} 2 \mathrm{~A} \cdots \mathrm{O} 2$ & 2.52 & 3.397 & 149 & $x,-1+y, z$ \\
\hline
\end{tabular}




\begin{tabular}{|l|l|l|l|l|l|}
\hline & $\mathrm{C} 4-\mathrm{H} 4 \cdots \mathrm{O} 7$ & 2.59 & 3.262 & 129 & $-1+\mathrm{x},-1+\mathrm{y}, \mathrm{z}$ \\
\hline & $\mathrm{C} 16-\mathrm{H} 16 \mathrm{~A} \cdots \mathrm{O} 2$ & 2.46 & 2.830 & 102 & $\mathrm{x}, \mathrm{y}, \mathrm{z}$ \\
\hline & $\mathrm{C} 23-\mathrm{H} 23 \mathrm{~A} \cdots \mathrm{O} 4$ & 2.55 & 3.460 & 155 & $\mathrm{x}, 1+\mathrm{y}, \mathrm{z}$ \\
\hline & $\mathrm{C} 37-\mathrm{H} 37 \mathrm{~A} \cdots \mathrm{O} 4$ & 2.54 & 2.874 & 100 & $\mathrm{x}, \mathrm{y}, \mathrm{z}$ \\
\hline & $\mathrm{C} 54-\mathrm{H} 54 \cdots \mathrm{O} 8$ & 2.55 & 3.216 & 128 & $-1+\mathrm{x}, \mathrm{y}, \mathrm{z}$ \\
\hline
\end{tabular}

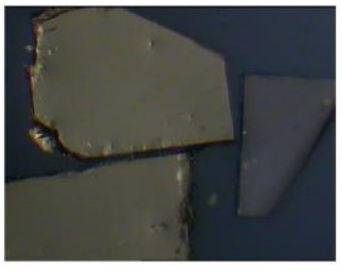

(a)

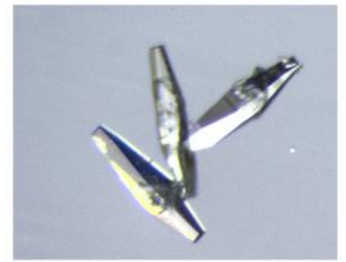

(c)

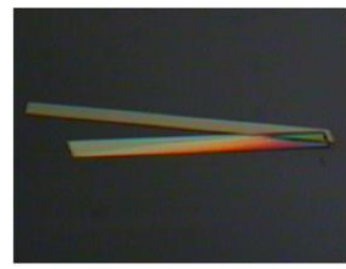

(b)

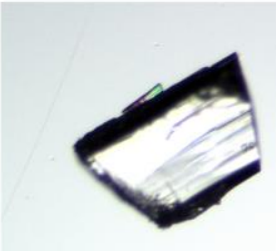

(d)

Figure S2. Polarizing microscopy pictures for cocrystals: (a) Prog-SPF, (b) Prog-SPFHH, (c) Prog-RSV, (d) Prog-RSV-HH.

Table S2. Thermal Data of Prog and Cocrystals

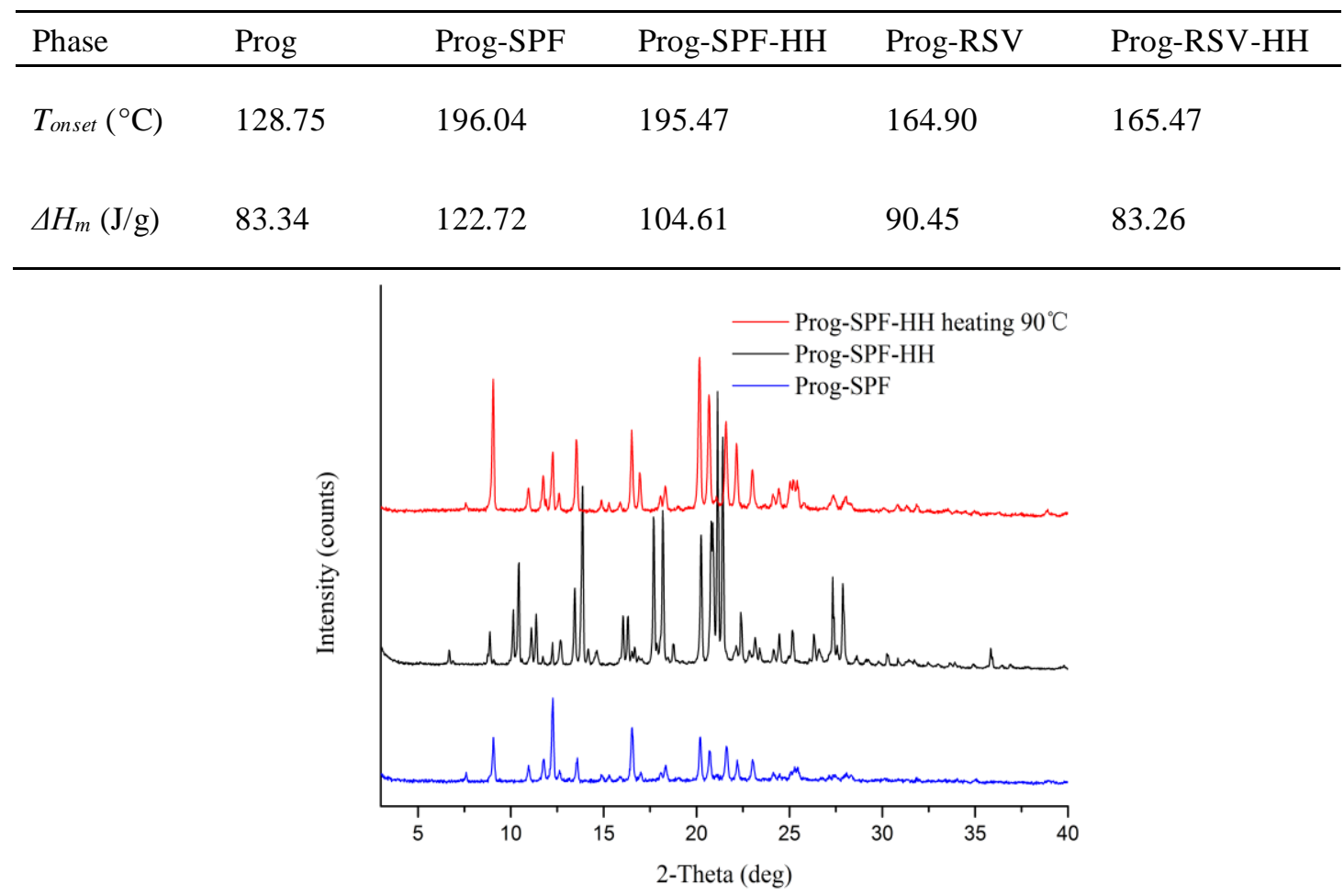


Figure S3. The VT-PXRD patterns of Prog-SPF-HH.

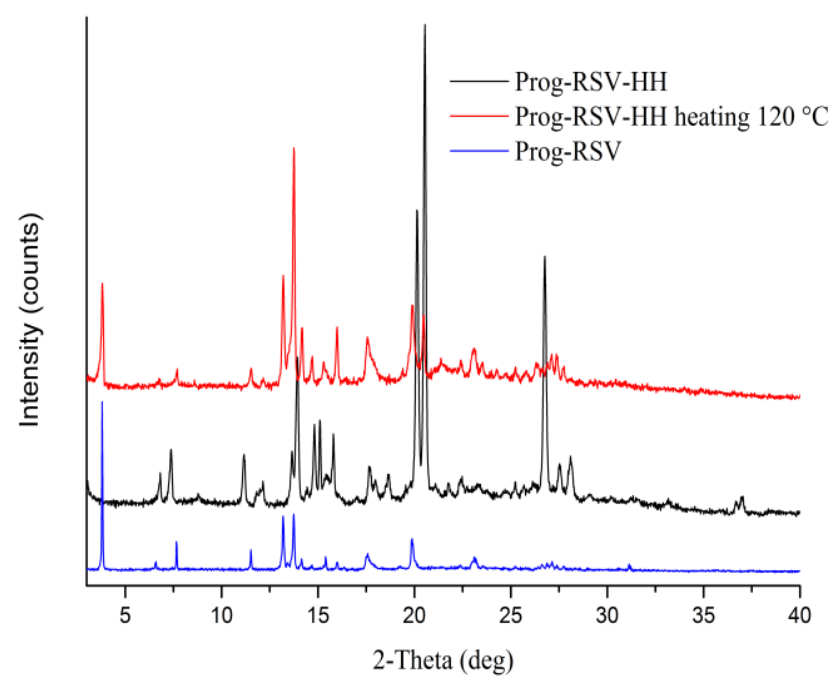

Figure S4. The VT-PXRD patterns of Prog-RSV-HH.

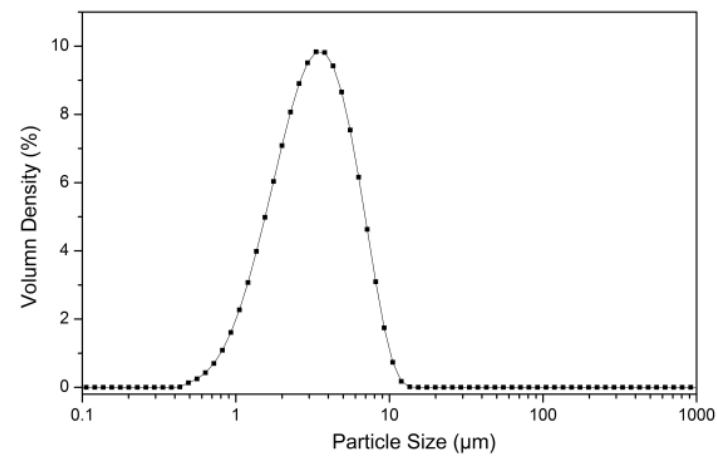

Figure S5. Particle size distribution for micronized Prog (measured by Malvern Mastersizer 3000).

Table S3. The Maximum Concentrations and Reached Times

\begin{tabular}{lllll}
\hline \multirow{2}{*}{ Surfactant } & $1 \%$ & $0.5 \%$ & $0.5 \%$ & $0.5 \%$ \\
& HPC & STFBB & Tween 80 & PVP-K30 \\
\hline
\end{tabular}




\begin{tabular}{lllll}
\hline $\mathrm{C}_{\max }$ of Prog $(\mu \mathrm{g} / \mathrm{mL})$ & 16 & 22 & 69 & 14 \\
$\mathrm{t}_{\max }$ of Prog $(\mathrm{min})$ & 25 & 25 & 10 & 10 \\
\hline $\mathrm{C}_{\max }$ of Prog-SPF $(\mu \mathrm{g} / \mathrm{mL})$ & 27 & 36 & 124 & 24 \\
$\mathrm{t}_{\max }$ of Prog-SPF (min) & 20 & 20 & 10 & 5 \\
$\mathrm{C}_{\max }$ Prog-SPF / Cmax Prog & 1.7 & 1.6 & 1.8 & 1.7 \\
\hline $\mathrm{C}_{\max }$ of Prog-RSV ( $\left.\mu \mathrm{g} / \mathrm{mL}\right)$ & 9 & 10 & 36 & 16 \\
$\mathrm{tm}_{\max }$ of Prog-RSV (min) & 5 & 10 & 5 & 5 \\
$\mathrm{C}_{\max }$ Prog-RSV / C $\max$ Prog & 0.6 & 0.5 & 0.5 & 1.1 \\
\hline
\end{tabular}
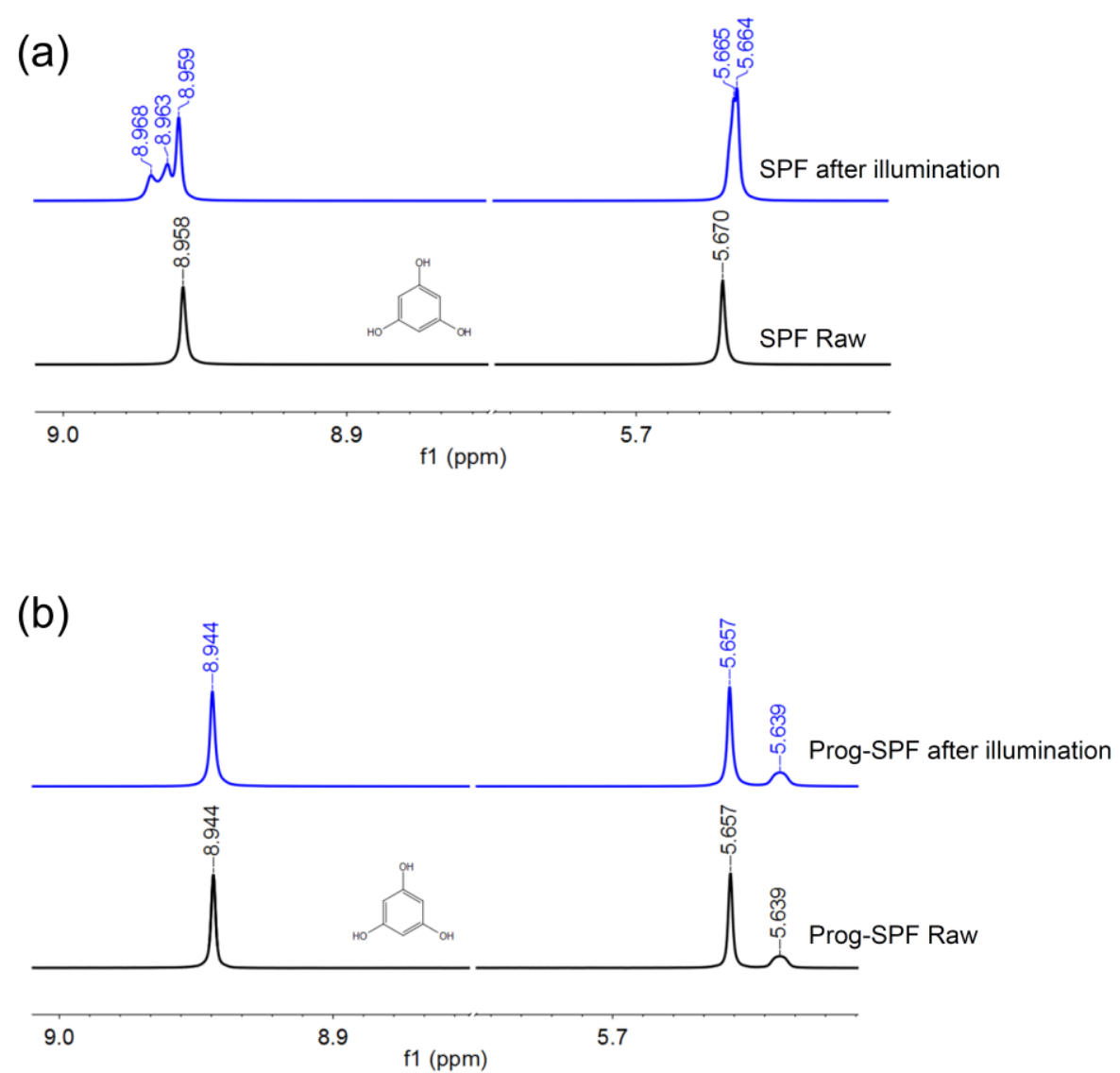

Figure S6. The overlays of ${ }^{1} \mathrm{H}-\mathrm{NMR}$ spectra of (a) SPF and SPF after illumination,

(b) Prog-SPF and Prog-SPF after illumination. 


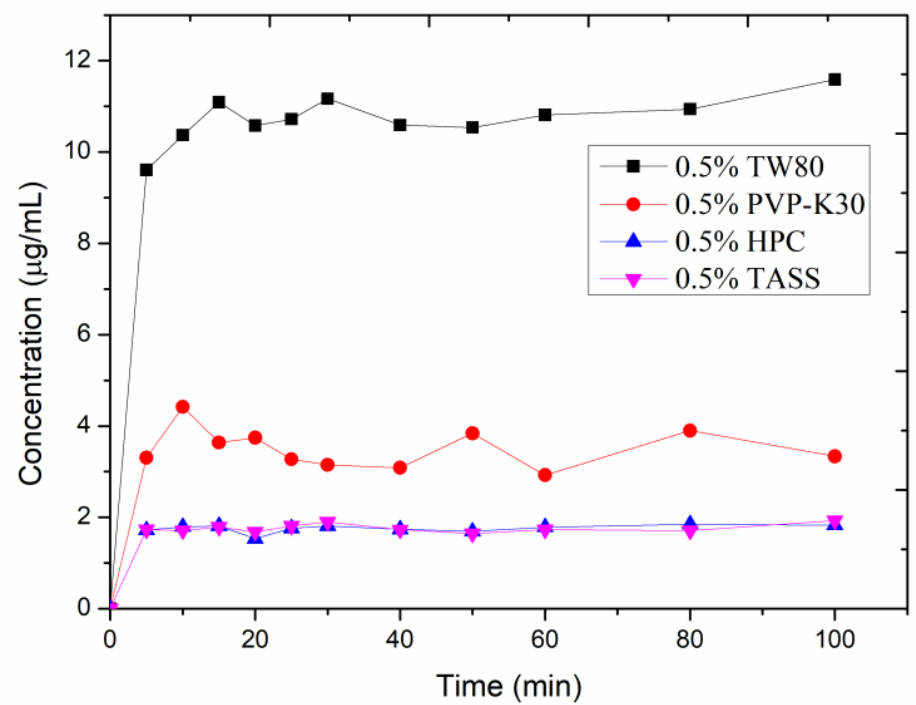

Figure S7. Powder dissolution profiles of Prog-RSV-HH. 